\title{
Lateral pterygoid muscle dystonia. A new technique for treatment with botulinum toxin guided by electromyography and arthroscopy
}

\author{
Pedro Martos-Díaz ${ }^{1}$, Francisco-José Rodríguez-Campo ${ }^{2}$, Raquel Bances-del Castillo ${ }^{3}$, Oscar Altura-Guillén ${ }^{4}$, \\ Gui-Youn Cho-Lee ${ }^{5}$, María Mancha-de-la Plata ${ }^{6}$, Veronica Escorial-Hernandez ${ }^{7}$
}

\begin{abstract}
${ }^{1}$ Assistant Physician, Department of Maxillofacial Surgery. Hospital Universitario Nuestra Señora de la Candelaria. Tenerife ${ }^{2}$ Assistant Physician, Department of Maxillofacial Surgery. Hospital Universitario de La Princesa. Madrid

${ }^{3}$ Assistant Physician, Physical Medicine and Rehabilitation. Hospital Universitario Nuestra Señora de la Candelaria. Tenerife

${ }^{4}$ Assistant Physician, Department of Maxillofacial Surgery. Hospital Universitario Nuestra Señora de la Candelaria. Tenerife

${ }^{5}$ Assistant Physician, Department of Maxillofacial Surgery. Hospital Universitari Clinic. Barcelona

${ }^{6}$ Assistant Physician, Department of Maxillofacial Surgery. Hospital Montepríncipe. Madrid

${ }^{7}$ Assistant Physician, Department of Maxillofacial Surgery. Hospital Universitario de La Princesa. Madrid
\end{abstract}

Correspondence:

Departamento de Cirugía Maxilofacial,

Hospital Universitario Nuestra Señora de la Candelaria,

Santa Cruz de Tenerife,

C/ Carretera del Rosario $S / N$,

Santa Cruz de Tenerife, Spain

plmartos9@hotmail.com

\begin{abstract}
Martos-Díaz P, Rodríguez-Campo FJ, Bances-Del Castillo R, AlturaGuillén O, Cho-Lee GY, Mancha-de la-Plata M, Escorial-Hernandez V. Lateral pterygoid muscle dystonia. A new technique for treatment with botulinum toxin guided by electromyography and arthroscopy. Med Oral Patol Oral Cir Bucal. 2011 Jan 1;16 (1):e96-9.

http://www.medicinaoral.com/medoralfree01/v16i1/medoralv16ilp96.pdf
\end{abstract}

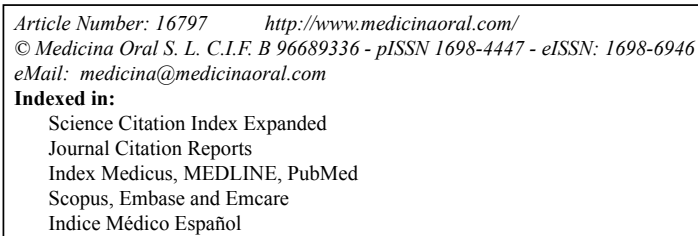

\begin{abstract}
Lateral pterygoid muscle dystonia is characterized by mandibular displacement towards the opposite side of the affected muscle. It may be associated with functional disorders affecting speech, swallowing, chewing and facial symmetry. Injection with botulinum toxin is recognized as the most effective treatment. Locating the lower head of the lateral pterygoid muscle for the injection is not difficult using electromyographic guidance; however, location of the upper head is more complicated, even with electromyography. We report a case of lateral pterygoid muscle dystonia in which precise injection of the upper head was achieved with the aid of arthroscopy.
\end{abstract}

Key words: Oromandibular dystonia, lateral pterygoid, botulinum toxin, TMJ arthroscopy.

\section{Introduction}

Oromandibular dystonia (OMD) is characterized by prolonged contraction of one or more of the muscle groups encompassing the tongue, masticatory and facial muscles $(1,2)$. The result is abnormal posture, often uncomfortable and painful, leading to functional abnormalities that affect speech, swallowing, chewing and facial symmetry (3).
Lateral pterygoid muscle (LPM) dystonia, is described as focal OMD with jaw displacement.

The treatment of choice for OMD is injection of the affected muscles with botulinum toxin (4-6). In this regard, the LPM is problematic since location of the muscle requires adjuvant procedures in order to achieve precise access. 
A case of focal LPM dystonia is presented. We describe the presentation, clinical history, electromyographic (EMG) study and treatment with botulinum toxin injections guided by EMG for the lower head, and arthroscopy for the upper head.

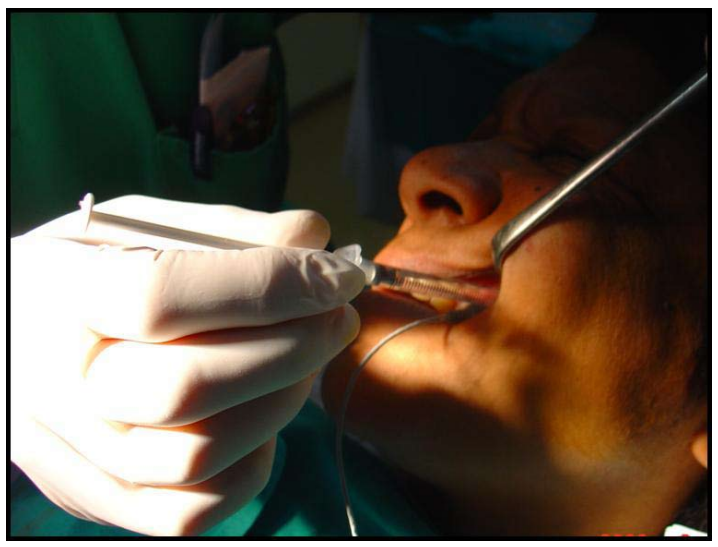

Fig. 2. Injecting lower head of LPM guided by EMG.

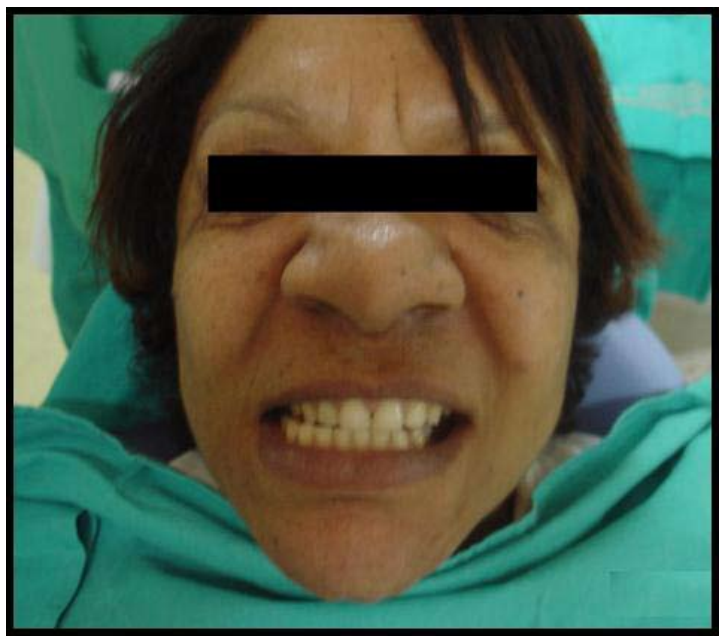

Fig. 1. LPM dystonia. Mandibular displacement to the contralateral side.

\section{Case Report}

A 47-year-old woman, following idiopathic facial paralysis began having difficulties with chewing and speech. The facial paralysis had been treated with oral corticosteroids, resolving at 4-5 months of presentation.

Two years later the patient attended our clinic, reporting that since the previous treatment she had continued to have difficulties with speech, swallowing and chewing. With the mouth closed, a mandibular displacement of $7 \mathrm{~mm}$ to the right side was apparent (Fig. 1). With the mouth open the dental midlines were centered. Using external manual aid a centric relation was obtained at maximum intercuspal occlusion. However, when the manual aid was withdrawn the initial displacement reappeared.

An EMG study was conducted using the Sapphire electromyograph $4 \mathrm{Medelec}^{\circledR}$. On puncturing the lower head of the LPM on the left side, an increase in activity at mandibular rest was observed, confirming the clinical suspicion.

Having confirmed the diagnosis of OMD of the LPM, the appropriate dilution for injection of botulinum toxin was prepared. Thus, 100 units of type A toxin (Botox ${ }^{\circledR}$ ) were diluted in $4 \mathrm{cc}$ of saline solution, obtaining 20 units, which were injected intraorally into the lower head using EMG guidance (Fig. 2).

One month after injection, an improvement in speech and swallowing was observed. Mandibular displacement had improved, although a displacement of $4 \mathrm{~mm}$ still remained. It was decided to complement the treatment by infiltrating the upper head. Firstly, a left TMJ arthroscopy was made using a Stryker ${ }^{\circledR}$ arthroscope with a 1.9 $\mathrm{mm}$ optic and 30 degree angulation. On locating the upper head of the LPM in the anterosuperior compartment of the joint, a second trocar was introduced using the triangulation technique. Using a 27-Gaus needle the upper head of the LPM was then punctured and infiltrated with another 20 units of botulinum toxin at the same dilution as before (Fig. 3).

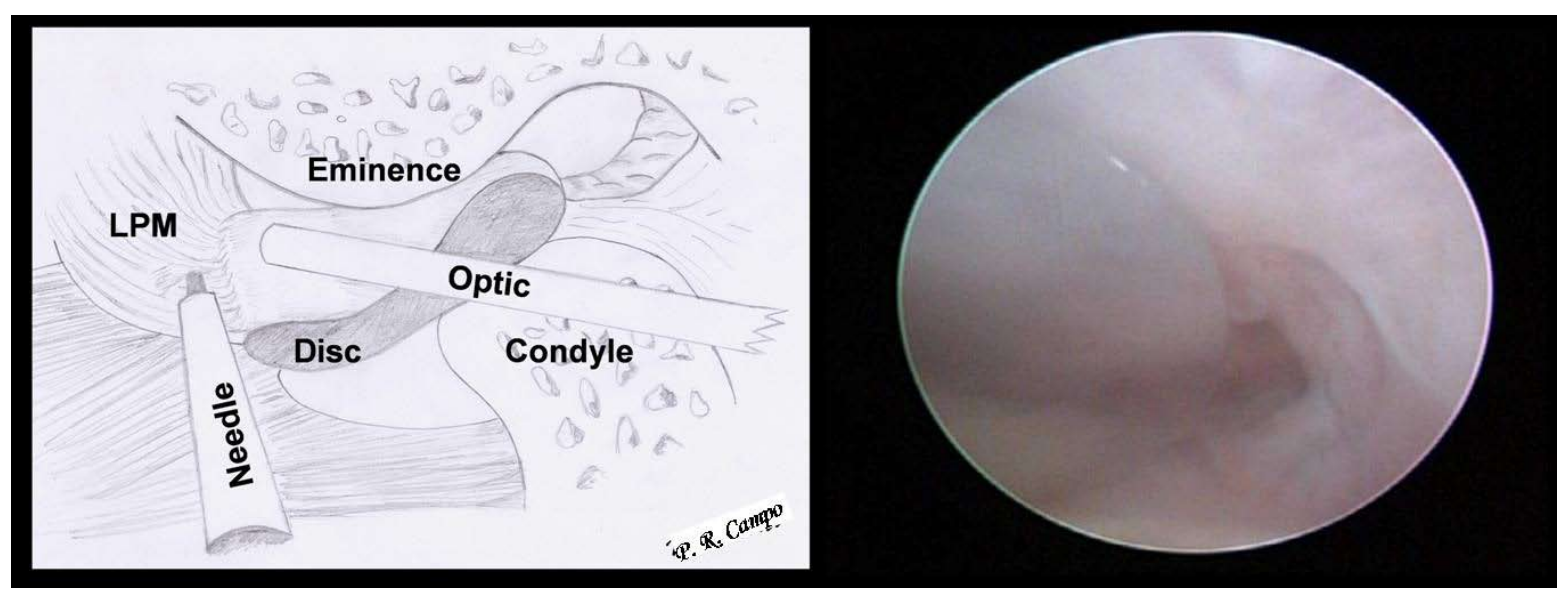

Fig. 3. Schematic view and arthroscopic image. Intraarticular, anterosuperior capsule of the left TMJ, with the needle passing through the trocar and infiltrating the upper head of the LPM. 


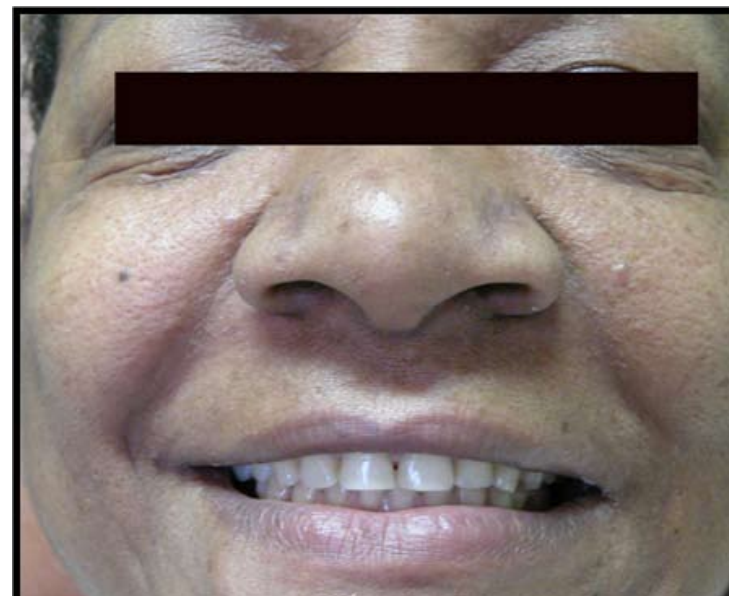

Fig. 4. Patient after injection of both LPM heads with botulinum toxin.

The patient was examined 20 days after treatment, and again at 3 and 6 months. In the first 3 months the patient was able to achieve a correct occlusion with no external assistance and with no dental displacement (Fig. 4). However, at the six months review, the mandibular displacement had returned to virtually the same condition as prior to the injections. Subsequent injections were made only in the lower head of the LPM, obtaining only partial results.

\section{Discussion}

OMD can affect different muscle groups. It is important to be able to recognize and locate these muscles in order to make the selective treatment.

Few data exist in the literature on diagnosis and treatment of focal LPM dystonia, (7). This disorder is easily confused with other conditions involving mandibular asymmetries, such as TMJ dysfunction, and occlusal or mandibular bone abnormalities.

The most characteristic sign is mandibular displacement to the contralateral side of the affected muscle, and may be associated with pain, occlusal disorders, difficulties in speech and, swallowing, aesthetic alterations, and psychosocial disorders (3).

The LPM consists of two heads. The upper head arises from the greater wing of the sphenoid and inserts onto the articular disc and capsule of the temporomandibular joint; while the lower arises from the lateral aspect of the lateral pterygoid plate and inserts into the neck of the mandibular condyle. Classically the upper head is primarily associated with mouth opening movements while the lower head is related to jaw closing movements. Bilateral contraction of the LPM produces a protrusive mandibular movement. Unilateral contraction produces a mandibular displacement to the contralateral side of the contracted muscle. In electromyographic studies various authors have found activity in both LPM heads during lateral mandibular displacement (in the LPM contralateral to the movement), and in mandibular protrusion (in both LPM) (8).

Several studies support botulinum toxin injection of the affected muscles as the treatment of choice for OMD $(4,6,9,10)$. Most authors report varying degrees of improvement in symptoms when injecting the drug either directly or guided by EMG (11). This improvement was also obtained in our patient following injection of the lower head guided by EMG; however the lateral mandibular displacement persisted, albeit to a lesser degree. We associate the lack of complete response to injection of the lower head alone, without injecting the upper head.

Although locating the lower head for treatment is not complicated, locating the upper head is difficult, even with the help of EMG. Since the upper head inserts at the level of the disc-condyle complex, one possibility would be to locate this at intraarticular level in the anterosuperior compartment. This can be accessed through the pterygoid window with little difficulty, although always under direct vision aided by an arthroscope.

This article describes the only case in the literature where accurate injection of the upper head of the LPM is achieved through guidance with the aid of arthroscopy of the TMJ.

TMJ arthroscopy has a low morbidity for the patient and has proven effective in cases of TMJ dysfunction with a very low percentage of complications $(12,13)$. Furthermore, as can be seen, the procedure can be very helpful in locating the upper head of the LPM. For this reason, although invasive, arthroscopy could be considered in specific cases of severe dystonia of this muscle with significant functional impairment that does not improve after direct injection of the lower head alone.

\section{Conclusion}

When treating OMD, it is important to make an accurate diagnosis of the muscles involved to facilitate effective treatment by injection of botulinum toxin. In this report, in addition to proposing a new treatment option for severe LPM dystonia by arthroscopy of the TMJ, we corroborate the clinical involvement of both heads of the LPM in lateral mandibular displacements. Therefore, in LPM dystonia, injection of botulinum toxin in both heads should be considered in order to obtain a complete response.

\section{References}

References with links to Crossref - DOI

1. Jankovic J, Ford J. Blepharospasm and orofacial-cervical dystonia: clinical and pharmacological findings in 100 patients. Ann Neurol. 1983;13:402-11.

2. Yoshida K, Kaji R, Kubori T, Kohara N, Iizuka T, Kimura J. Muscle afferent block for the treatment of oromandibular dystonia. Mov Disord. 1998;13:699-705. 
3. Bhattacharyya N, Tarsy D. Impact on quality of life of botulinum toxin treatments for spasmodic dysphonia and oromandibular dystonia. Arch Otolaryngol Head Neck Surg. 2001;127:389-92.

4. Blitzer A, Brin MF, Greene PE, Fahn S. Botulinum toxin injection for the treatment of oromandibular dystonia. Ann Otol Rhinol Laryngol. 1989;98:93-7.

5. Blitzer A, Sulica L. Botulinum toxin: basic science and clinical uses in otolaryngology. Laryngoscope. 2001;111:218-26.

6. Tan EK, Jankovic J. Botulinum toxin A in patients with oromandibular dystonia: long-term follow-up. Neurology. 1999;53:2102-7.

7. Yoshida K, Kaji R, Takagi A, Iizuka T. Customized EMG needle insertion guide for the muscle afferent block of jaw-deviation and jaw-opening dystonias. Oral Surg Oral Med Oral Pathol Oral Radiol Endod. 1999;88:664-9.

8. Murray GM, Orfanos T, Chan JY, Wanigaratne K, Klineberg IJ. Electromyographic activity of the human lateral pterygoid muscle during contralateral and protrusive jaw movements. Arch Oral Biol. 1999;44:269-85.

9. Blitzer A, Brin MF, Greene PE, Fahn S. Botulinum toxin injection for the treatment of oromandibular dystonia. Ann Otol Rhinol Laryngol. 1989;98:93-7.

10. Møller E, Bakke M, Dalager T, Werdelin LM. Oromandibular dystonia involving the lateral pterygoid muscles: four cases with different complexity. Mov Disord. 2007;22:785-90.

11. Mendes RA, Upton LG. Management of dystonia of the lateral pterygoid muscle with botulinum toxin A. Br J Oral Maxillofac Surg. 2009;47:481-3.

12. Mancha de la Plata M, Muñoz-Guerra M, Escorial Hernandez V, Martos Diaz P, Gil-Diez Usandizaga JL, Rodriguez-Campo FJ. Unsuccessful temporomandibular joint arthroscopy: is a second arthroscopy an acceptable alternative? J Oral Maxillofac Surg. 2008;66:2086-92.

13. González-García R, Rodríguez-Campo FJ, Escorial-Hernández V, Muñoz-Guerra MF, Sastre-Pérez J, Naval-Gías L, et al. Complications of temporomandibular joint arthroscopy: a retrospective analytic study of 670 arthroscopic procedures. J Oral Maxillofac Surg. 2006;64:1587-91. 